\title{
11 Conclusions from case studies and survey
}

\author{
Saskia Tjepkema
}

The case studies, reported in Chapters 4 to 10 , were followed by a survey of a 165 companies in the seven participating countries. The purpose of the survey was to investigate whether findings from the case studies could be considered representative for a larger number of learning-oriented organisations throughout Europe. Since the survey results tended to corroborate the case study outcomes, in this chapter, the main conclusions from both are discussed jointly. The chapter provides a general account of the results, focusing on 'content' outcomes, rather than on numerical results. Readers interested in the specifics of the case and survey results, are referred to the original project reports (Tjepkema, et al., 1999; Tjepkema et al., 2000).

The study set out to answer three main research questions:

1 How do HRD departments in learning-oriented organisations throughout Europe envision their own role in stimulating and supporting employees to learn continuously as a part of everyday work (with the intention of contributing to organisational learning, and thus enhancing organisational competitiveness)?

2 What strategies do European HRD departments adopt to realise their envisioned role?

3 What factors inhibit the realisation of this new role? How do HRD practitioners cope with these factors?

4 What factors facilitate the realisation of HRD's new role?

The main results from the case studies and the survey are provided for each of these questions. First, it is important to point out that from these data, it is not possible to make judgements concerning the situation in any specific country, because the project only incorporated some 'good examples' of learning-oriented organisations from each participating EU nation. The researchers did not select a representative group of companies, since this is not possible in either case studies, or small-scale survey research. It is impossible to make a judgement on the situation in a specific country based on only a few cases, but the data were checked for national differences nonetheless. In general no significant differences were found between the situation in the seven participating countries. 


\section{Organisational context}

Before describing results for the actual research questions it is important to briefly address the organisational context. This section therefore examines issues such as: the firms' main strategic business issues, reasons for wanting to increase their potential for organisational learning, and main change strategies employed in pursuing this goal.

\section{Business strategy}

It was found that all twenty-eight cases, as well as almost all of the 165 organisations participating in the survey, are currently facing strong(er) competitive markets (caused for instance by globalisation) and/or fast(er) changing technologies. As a result, improving and innovating products, processes and/or services and increasing client centredness were found to be key elements in most business strategies. These strategies have in common that they require great flexibility, since this enables the organisation to respond quickly to, for instance, technological innovations or shifting client needs. Some organisations even explicitly adopted strategies directly related to increasing flexibility, for example reducing delivery times.

Human resources were mentioned by almost all of the case organisations as well as the survey participants as an important means to realise these business objectives. People are regarded as a key asset in meeting the main strategic challenges, such as increasing client focus, innovation, improvement and flexibility. While business is inevitably becoming more technological, paradoxically it is people that are becoming the key to competitiveness (see also: Lifelong learning for European Business, 1993). Employee learning and related issues, such as knowledge management/knowledge sharing and creating a learning culture were found to be key issues for most organisations in this study.

By the way, not all case organisations officially stated that they intend to become a learning organisation. Nevertheless, most firms appear to be developing towards a learning organisation (by means of, for instance, improving employee learning and working on a learning culture) without explicitly stating this as a major organisational goal. Becoming a learning (oriented) organisation is not so much a strategic business objective itself, but rather a way to realise such strategic business objectives, and as such a response to an increasingly turbulent business environment (increased competition, technological advances, etc).

Though a strategic intent to become more client-centred, among other things by realising on-going improvement and innovation, and greater flexibility, seems to be a main motivator for wanting to become a learning organisation, more people-oriented reasons appear to play a role as well. Such reasons include, for instance:

- a lack of vocational training which forces the company to create learning opportunities for staff (in two of the French cases, see Chapter 6);

- the intention to retain employees and/or reduce employee turnover (which is the case for instance in the Belgian cleaning company ISS, see Chapter 4);

- increasing quality of working life. 


\section{Change processes}

The organisations in this study were found to adopt a large variety of change strategies, in order to realise their (new) strategic goals. Change processes most commonly addressed topics such as:

- creating a client-oriented culture;

- changing the management style;

- improving the strategy development process (e.g. creating a shared mission statement);

- changing the structure (e.g. implementing team working);

- optimising (IT) systems;

- creating a learning culture;

- $\quad$ supporting employee development.

Given the outlook of this study, those change strategies focusing on creating a learning culture and on employee development are of greatest interest. Creating more learning opportunities, improving worker motivation for learning and supporting a continuous development process for employees constitute examples of change initiatives directed at development of a learning culture. Examples of activities targeted at employee development include the implementation of competence management and personal development plans. It is interesting to note that organisations appear to focus on the role of human resources in the organisation, in general. They don't seem to limit themselves to employee development but also use other HR initiatives and instruments, such as selection and recruitment, and job rotation.

In general, companies employ a rich array of change initiatives, in which no one type of change is particularly dominant. Which types of changes are initiated in any given firm is probably determined by a mix of factors, such as strategic business objectives, employee characteristics, current organisational problems, organisational structure and management style. Untangling these relationships falls beyond the scope of this project, which focuses mainly on HRD, not on the companies as a whole.

One final observation is worth mentioning here. Though a common theme throughout the research has been the motivation for becoming more learning-oriented, in order to realise improvements in business performance and competitive advantage, this seemingly causal relationship does not seem to be extensively evaluated in any of the organisations. In other words, no one has looked for 'proof' that increasing the organisational capacity for learning will indeed increase organisational effectiveness. Their trust in the concept of the learning organisation can therefore probably be explained as an act of faith, or as one of faithfully copying what apparently successful organisations seem to be doing.

\section{HRD's envisioned role}

The first research question was: how do HRD departments in learning-oriented organisations throughout Europe envision their own role in stimulating and supporting employees to learn continuously, as a part of everyday work? In order to come up 
with an answer, HRD objectives were investigated, as well as the 'HRD partnership': who are involved in HRD, and what roles do these different people assume? This section summarises the results.

\section{HRD objectives}

Survey results regarding important HRD objectives are highly similar to the case study outcomes. In general, objectives in four areas could be distinguished:

- $\quad$ supporting the business;

- $\quad$ supporting (informal) learning and/or supporting knowledge sharing;

- providing training;

- changing HRD practices.

\section{Supporting the business}

A first category of objectives is related to the strategic goal of 'supporting the business', either in a general sense, or by supporting specific current strategic company objectives. Examples of such business-oriented objectives are: 'helping the company to meet current and future challenges', 'making sure training contributes to corporate strategy' and 'keeping the organisational skill base up-to-date'. Or in other words, making sure the workforce possesses the necessary knowledge and skills to fulfil those tasks required to realise company objectives. This task encompasses activities such as analysing the existing skill base, analysing current and future competence requirements and recruiting and training employees to make sure these requirements are met. Related objectives are, therefore, 'defining key competence areas or organisational learning needs', 'helping to retain employees' and 'stimulating employee versatility'.

As well as objectives regarding support for the business in a general sense, some HRD functions mention specific organisational objectives which they seek to support. For instance: 'enhancing organisational flexibility', 'supporting managers in their new role', or 'supporting the customer orientation of the company'.

\section{Supporting (informal) learning and knowledge sharing}

A second set of objectives exists of goals regarding support for (informal) learning of employees and/or teams and the organisation as a whole. Within this category, three types of objectives can be distinguished.

First, objectives with regard to supporting employee learning in general, such as 'promoting employee development', 'contributing to learning', 'contributing to competence development', or 'creating conditions for lifelong learning'.

It is interesting to note the inherent tension between the desire to support the business that was mentioned earlier, and the objective to support individual learning. Linking these two is not always a straightforward task, as some case organisations also experience. For instance the Finnish paper machine manufacturer Valmet strives for a strong connection between training activities and business activities (see 
Chapter 5). In order to strengthen this link, once a year, development discussions are organised. In these meetings, employees' needs and possibilities for development are evaluated. But, according to managers, a connection to the organisational strategy is not always created. In using personal development plans, the focus is very much on personal learning issues. This is probably quite a widespread phenomenon.

A second group of objectives can be distinguished which express explicit support for informal learning, such as: 'implementing a learning culture', 'stimulating and supporting new knowledge creation', 'engaging and motivating employees in learning and working' and 'stimulating appreciation and use of informal learning opportunities on-the-job'. Of note here is the variation in terminology; whereas some companies use terms such as 'learning culture' and 'informal learning', others speak of 'knowledge creation'. This is probably largely a matter of terminology, but it might, however, also reflect a somewhat different outlook on the types of learning processes these firms concentrate their attention on. The term knowledge management appears to reflect a greater emphasis on sharing knowledge or making sure knowledge becomes available to more people in the organisation, whereas in the concept of the learning organisation, the focus seems to be more on creating opportunities for learning and development for individuals (and also for teams and the organisation as a whole).

Supporting learning does not seem to be simply a matter of providing opportunities for learning. Stimulating motivation for learning is a recurring issue in some of the objectives mentioned above (e.g. 'engage and motivate employees'). Also, attention to creating a broad perspective on learning can be detected. In general, most employees and managers (and even HRD professionals) hold a somewhat limited view on 'learning'. They usually (implicitly) equate it to classroom training. In some case organisations, the HRD professionals were found to actively try to establish a broader vision of learning. For instance, the British aero-engine manufacturer Rolls-Royce Airline Business, mentions it as an explicit objective (see Chapter 10). One of its HRD managers explained the relevance of creating a broader view on learning:

There is an immense amount of on-the-job learning. Many employees would not have been conscious of this form of learning. If you asked them to list what learning they had been involved in, they would think 'what course did I go on, what hotel did I stay in, what exam did I take?' Learning means doing the job, learning from colleagues, training others, doing a first aid certificate, having an enlarged job. Coaching is important - often the coach learns more than the coachee. Every new experience is an opportunity to learn.

Finally, both case study and survey evidence suggest that most companies do not focus exclusively on individual learning, but also aim to support team and even organisational learning. The emphasis, however, is on the individual level.

\section{Providing training}

Whereas informal learning does receive explicit attention, objectives with regard to providing training, such as 'development and co-ordination of training' and 'offering a supply of training activities' also form an important group. 
The objective of the French semi-conductor plant Motorola, which mentions that it seeks to centralise its training efforts in order to get a more global and accurate view and a better strategic control of the training investments (see Chapter 6) is worthy of note here. It seems that this is a necessary prerequisite for ensuring that training contributes effectively to the corporate strategy, which is HRD's main aim.

\section{Changing HRD practices/organisation of $H R D$}

In order to provide (informal) learning opportunities, and to provide a strategic contribution to the firm, some organisations have formulated objectives with regard to changing HRD practices or structures. Of course, this is an intermediary objective, it is not a purpose in itself, but something HRD functions pursue in order to better fulfil their main responsibilities. The most important objectives in this field are related to integrating HRD more strongly in the work organisation, for instance by sharing HRD tasks with line managers. Examples of these objectives include: 'providing services to/supporting management', 'working on a demand-oriented basis', 'stimulating management to become more sensitive to HRD issues' and 'integrate HRD in business functions'.

Other objectives, reflecting changing HRD practices are:

- adopting a more pro-active role as HRD department, for instance: 'changing from a reactive to a proactive role' and 'playing a leading role in the development of a new management system';

- adopting new work methods, for instance: 'realising/improving competence management';

- adopting a new approach to HRD, for instance: 'increase self-management in learning' and 'change from human resource control to human resource development'

\section{Most important objectives?}

Though it was attempted to establish one in the survey, differences in importance between these sets of objectives are too small to reflect a real rank order. Whichever objective is stressed most depends on the specific situation of each organisation. It is interesting to note though, that the expectation of survey respondents was that objectives in the field of supporting informal learning and promoting knowledge sharing are expected to increase most in importance in the future. It is also relevant to point out that supporting the business (objectives) is one of the most important HRD objectives, but survey results suggest that this involvement is usually not very great. So, the question as to how far HRD fulfils a strategic role remains open.

\section{People involved in HRD}

'Who does what in HRD?' With regard to the sharing of HRD tasks, it is clear that HRD professionals still carry the biggest share of responsibility for HRD (at least in 
their own estimation of the situation). Managers and employees are important active partners, and are expected to become more so in the future.

\section{HRD professionals}

In general, HRD professionals' work in the learning-oriented organisations in this study still largely consists of what we might call 'traditional' HRD tasks, such as developing, providing and/or co-ordinating training. This finding supports the assumption made in the previous section that training is still a significant part of the work field of HRD professionals in the learning-oriented organisations. Other 'basic' HR tasks such as giving advice to employees, for instance on career paths, and selection and recruitment were also mentioned.

Perhaps more interesting than the regular HRD tasks are the 'new' tasks, which were also found. In the vast majority of both the case and survey organisations, HRD professionals mentioned such non-traditional tasks as part of their work portfolio. The most important ones are: consulting to line management and providing tools and (practical) support with regard to learning issues to line managers (and employees).

Both tasks reflect a shift in the responsibility for learning towards managers - who are expected to assume a more active role in supporting their employees in learning as well as towards employees themselves. We will look more closely into both their roles below. As for the HRD professionals, if managers and employees take over some of the more practical HRD tasks, this means HRD professionals will have to support them in doing so, in order to ensure quality of HRD interventions. An effective way in which to give this support is to provide them with methods and tools (e.g. personal development plans) that stimulate self-management in learning. A good example is provided by British insurance agency Royal Scottish Assurance, where the HRD department has developed a competency development guide for employees, listing all the available resources for learning (see Chapter 10). This supportive role is in essence a rather practical one.

Having managers fulfil an active role in HRD also gives HRD practitioners room to give advice and to consult on HRD issues, which can be considered to be a more strategic role. Advice might be directed towards helping managers to link training to corporate needs, to increase and use opportunities for informal learning at work (for instance by coaching or working on a learning climate) and to monitor competencies in a team or department.

\section{Line managers}

As mentioned above, the supportive and consulting role of HRD professionals is consistent with the way in which HRD views the role of managers and employees. In HRD's envisioned role, management fulfils an active role in supporting employee learning.

Sometimes management is already held responsible for employee learning. Sometimes HRD practitioners indicate that management will carry that responsibility in the future. In practice this means that managers are, or will become, responsible for some 
of the practical HRD tasks, such as making individual development plans, monitoring competencies, defining learning needs, competence assessment, evaluation of training or even more strategic tasks, such as implementing HRD policy, implementing the learning organisation concept or fulfilling a steering role with regard to HRD.

An interesting exception to the rule is encountered in the Belgian cleaning company ISS (see Chapter 4). This company employs two HRD practitioners, who are responsible for the administration and logistic support of training programmes. Their role is very restricted and reactive, and consists primarily of providing employees with adequate and sufficient training possibilities. Responsibility for defining and implementing the concept of the learning organisation lies with top management, who define HRD policies. Finally, line management is responsible for HRD in the daily work environment. This situation is interesting because it clearly differs from that in most organisations in this study. In most cases, HRD professionals fulfil a more active or even strategic role in the development of their companies, though the degree of pro-activity differs from company to company. In some firms, HRD even serves as real change agents and a driving force in the development towards a learning organisation.

\section{Employees}

Employees are also expected to fulfil an active role in their own development. They either already have a responsibility for their own learning or will share that responsibility with management in the future. Though rarely mentioned, employees sometimes are even involved in providing training or fulfilling HRD tasks in general. The French retail organisation Auchan provides an interesting example of the latter (Chapter 6). Experienced and well-performing employees are selected and trained to become trainers for the new staff. An important aim of the employee/trainer system is to motivate newcomers to learn and develop, by setting the right example.

Two points are worth considering, given these results. The first is that there does not appear to be a difference between companies with a high degree of manual labour, and companies with a highly-educated workforce. Employees are being held responsible for their own learning both in companies from manufacturing industry, such as the Finnish metal factory Outokumpu Zinc, the Dutch chemicals plant Akzo Nobel and the British brewery Wolverhampton \& Dudley on the one hand, and in professional organisations such as the Finnish Okobank, the German consulting firm GTZ and the Dutch IT centre BAC on the other. Case study and survey results did not produce clear indications, but it would not be surprising if employee responsibility for learning is more difficult to achieve in production companies resembling Mintzberg's machine bureaucracy archetype (either currently or in the past), than in service industry companies that resemble professional bureaucracies. In the latter, employees traditionally already carry a large amount of responsibility for their own professional development (Mintzberg, 1979; van der Krogt, 1991).

A second point to be made is that the specific type of responsibilities to be taken on by employees seems to be less clear than for managers. For managers, responsibility for HRD means to fulfil several practical, or even strategic HRD tasks. For employees, 
the picture is not so clear. It appears that they are mainly expected to engage in active discussions with managers in analysing their learning needs or drawing up their personal development plans, and show an active and motivated attitude towards their own learning and development in general. In some organisations, they are also expected to act as trainer or mentor, or to engage in business improvement teams (where learning and working go hand in hand).

\section{External HRD professionals}

The survey revealed a fourth active partner in HRD, namely external institutions. Survey results indicate that these are also regularly involved in executing HRD tasks, though not on a large scale. Their most important task is (as could be expected) providing training. A large proportion of survey respondents lists them as being involved in this HRD task. Other relatively important tasks appear to be consulting on employee development and identification of employees with potential. External institutions are involved only very rarely in tasks such as making personal development plans, implementing HRD policy, promoting a learning culture, monitoring competencies and training needs analysis. These are all HRD tasks with a potentially strategic impact, which could be the reason why companies don't easily choose to outsource them.

\section{HRD as an integrated business function?}

When considering this description of the HRD professionals' new role, it is important to recognise that a gap may exist between HRD's self-image and vision and the expectations from the company. To give one example, according to HRD professionals from the Finnish Okobank, their role has changed already (see Chapter 5). Previously, they worked mostly as trainers, but now their main work consists of organising and co-ordinating personnel development functions. They try to clarify what the customers of the competence development unit need, maintain the flow of available training programmes, motivate and stimulate employees for learning, and create a learning culture. But according to line managers, there is still too much distance between the HRD unit and the business functions. Due to this, co-ordination between management and HRD is not yet clear and sufficient. Managers express an interest in more interaction, co-operation and new HRD initiatives. They hope for a more consultative role from HRD professionals and claim they want support in their responsibility for learning and development. Thus, it appears that the role of HRD professionals is changing, but still needs attention in this company. This probably also holds true for many more of the organisations in this study.

Though most HRD functions have not yet reached that point, it seems justified to conclude that the organisation of the HRD function is becoming more and more diffuse, and increasingly hard to identify and describe as a separate unit, as HRD is becoming ever more integrated within the business. This integration process appears to take place both with regard to policy-making (HRD policy linked more closely to, and eventually integrated in general strategy) and with regard to the execution of 
HRD activities (from being performed by HRD department, to a shared responsibility of HRD professionals, managers and employees). The competence managers, active in the research and development division of the Dutch branch of telecommunication company Ericsson (Chapter 9) form an example of a high degree of integration. There are now three types of managers in this company: operational managers, process owners (who are responsible for process management) and competence managers (responsible for people management). The latter operate as a sort of internal job agencies: at the start of each new project, they see ensure that each project has the necessary competencies (i.e. the necessary employees) to fulfil its objectives. Competence managers are part of the HRD function. Besides supporting employees with developing personal development plans and career development plans, they are also responsible for analysing required competencies in the organisation now and in the future.

Barham and Rassam (1989) describe this transition towards HRD integration as a 'shift from a fragmented or formalised approach to a focused approach on HRD', which they predicted to emerge in ever more companies. In the fragmented approach, training is a peripheral activity for the organisation. Training is seen as a very separate functional activity, managed exclusively by training professionals. The organisation is 'offered' training packages, in a manner which is usually described as the cafeteria approach: the HRD department lists the available training supply in a training catalogue, from which managers and employees then pick their courses.

Companies whose training efforts were fragmented in the past may decide to put more resources into training and make the whole process more systematic. This approach is described by Barham and Rassam as the formalised approach, because the training system is often linked into organisational systems (e.g. the appraisal system) to ensure that training takes place on a regular basis, and is linked to business needs.

Under the focused approach, training and development are still much more closely integrated in the organisation. As Barham and Rassam describe it:

It is [an approach] in which training is part of the lifeblood of the company, rather than being seen as a luxury or a dubious accessory. These firms are beginning to centre their training on three requirements: strategic business objectives, specific departmental needs and individual aspirations. Moreover, a recurring message is that the line managers themselves are taking a lead in directing training, rather than, as in the past, having to accept schemes devised by isolated training specialists. These companies are making a conscious attempt to root their training in what the organisation and its people actually need.

(Barham and Rassam, 1989: 122)

According to these authors, the roles of trainers in this approach broaden to include those of advisers, consultants, providers of resources and facilitators of learning (as opposed to 'directive interventionists'). They go on to explain that companies who use training and development in this way see continuous learning by individuals as a necessity, as part of their competitive strength. They see both off-the job training and work itself as opportunities to learn. As Barham and Rassam (1989) explain: 'they 
don't fall for the artificial distinction between work and learning', but instead recognise that most people are learning all the time. Support is needed for all learning activities, from formal off-the-job training to informal learning on-the-job. Based on the results described in this section, it can be concluded that most companies from this study are all making progress towards this focused approach to HRD.

\section{HRD strategies}

In the preceding section, a picture was sketched of the envisioned role of the HRD professionals. The image that emerged was one of an HRD function that aims to actively support the business by providing training and other opportunities for (informal) learning and knowledge sharing, so that the organisational knowledge and skill base is up-to-date and appropriate for realising the strategic objectives. HRD professionals, managers and employees are all active partners in this HRD function, it is not limited to an HRD department.

This section takes a closer look at the strategies adopted by the HRD professionals to support learning in their businesses, and more specifically, to achieve their objectives. In other words, this section provides an answer to the second research question: what strategies do European HRD departments adopt to realise their envisioned role?

Those HRD strategies encountered in both the case studies and the survey, can be categorised according to the main HRD objectives, described in the section on HRD's envisioned role. So, four sets of strategies could be discerned, with regard to:

- supporting the business in general, or supporting current strategic business objectives;

- supporting (informal) learning and knowledge sharing;

- providing training;

- changing HRD practices/structures.

\section{Support business in general/support current strategic objectives}

A first category of strategies was directed towards ensuring that HRD activities support the business (either in general, or with regard to a specific current strategic objective). An interesting example of such a strategy is having a strategic approach to training. The French semiconductor plant Motorola for instance, is moving from a catalogue approach to training, towards an approach in which contact with management is sought to ensure a solid link between training and company needs (see Chapter 6). Likewise, German chemicals manufacturer AgrEvo has also moved away from using a standard catalogue of traditional off-the-job training programmes towards an orientation on corporate objectives. To this end, it has established a competence management system and executes a strategic training needs analysis (see Chapter 7).

The second type of strategies is aimed not at supporting the business as a whole, but directed at specific strategic business objectives, such as implementing teamwork or changing the organisational culture. For instance, the Italian food producer Barilla supports the implementation and diffusion of self-designing work teams in 
manufacturing by specific interventions aimed at reinforcing the already acquired results and by presenting them to different areas, where line managers decide to start implementation. The interventions are of a step-by-step kind, steered by the specific needs managers have at any given time. Training programmes are being used, but so are other learning interventions (see Chapter 8).

A third type of strategies is formed by the use of HR instruments to ensure an adequate mix of competencies on the level of the organisation, thereby contributing to the organisation's competence level. In particular, competence management and applying new criteria in recruitment were mentioned. Both can be used as instruments to ensure the organisational skill base meets requirements. For instance, the Finnish steel producer Outokumpu Zinc has included a willingness to learn as a criterion in recruiting new staff. They use this as a means to create a staff that is motivated for learning (see Chapter 5).

\section{Support (informal) learning and knowledge sharing}

As well as formal training, which remains an important strategy, other methods to support (informal) learning were also found. The first cluster of strategies that can be distinguished consists of those employed to support (informal) learning from each other and knowledge sharing. Examples of such strategies include coaching, mentoring, learning groups, working on a variety of projects/secondments, and benchmarking. With regard to the latter, an interesting initiative was found at the British postal services, Royal Mail. This firm has installed a programme called 'Pathfinders', a benchmarking and problem-solving exercise for front-line employees. Eight employees are selected from each division, on an open and competitive basis, to form a team to examine a 'real' business problem and suggest improvements against a chosen business approach. The business problem is sponsored or championed by an operational manager, who is involved in helping the team. This is an illustration of a project that integrates learning and working, and stimulates employees to learn from each other (see Chapter 10).

An example of a learning group can be found in Sony Germany, where 'learning networks' are established. These operate in a way that resembles 'Action Learning'. Employees, who have at least one job element in common (e.g the same project, similar functions in different parts of the company) meet regularly to identify learning needs, and to develop competencies jointly, usually while working on a real business issue (see Chapter 7).

Strategies inspired by the theme of knowledge management were also found: for instance creating a knowledge database or building knowledge exchange networks. One organisation, the Dutch IT service provider BAC, has specifically investigated the possibilities for implementing knowledge management in its company (see Chapter 9). Technology is sometimes used explicitly to support knowledge sharing. For instance, the German manufacturer of household equipment, Bosch Siemens, and technical consulting firm GTZ have both developed information technology (IT) networks, aimed at facilitating and improving learning processes within the company. It is 
important to note, however, that these constitute corporate projects, and not HRD initiatives.

Another cluster of strategies to be discerned focuses on fostering employee responsibility for learning. Instruments and work methods such as personal development plans, self-directed learning, Open Learning Centres and learner-oriented learning methods all serve to increase employee self-management with regard to learning. An interesting example was found at the Finnish paper machine manufacturer Valmet (see Chapter 5). As an alternative to a traditional language course, Finnish participants who normally do not work as a team, were sent off to visit German clients. During this trip, speaking in Finnish was banned. In this way, participants developed international relationships, while at the same time improving their German language skills. This way of working is illustrative of a learning initiative where employees have a great influence on the actual programme and in which informal learning opportunities are deliberately created.

It needs to be recognised that HRD professionals sometimes also deliberately use informal learning methods as a way to change current notions on 'learning'. For instance, the HRD department at the British brewery Wolverhampton \& Dudley is keen to utilise a wide range of non-course-based methods such as coaching, mentoring, visits and secondments next to the more formal courses, to encourage managers and employees to change their perceptions of training and development from associating it with 'courses' to being willing and able to link work and learning (see Chapter 10). An example of this is an approach called 'three in a car'. Here, a trainer coaches managers to improve their coaching skills by observing and providing feedback to a manager coaching one of their staff.

Though the strategies mentioned above often also stimulate group learning, a third type of strategy could be found, aimed specifically at supporting team or even organisational learning (mentioned six times). For instance, at the British insurance company Royal Scottish Assurance (RSA), HRD professionals participate in the design and conduct of national and regional sales meetings, in order to facilitate and influence team and organisational learning. Learning that may emerge from the experiences of an individual worker will be shared with his or her colleagues through regional sales meetings. This will be passed on by an HRD practitioner to other training staff, who in turn will disseminate it in their regions (see Chapter 10).

\section{Providing training}

A substantial amount of HRD professionals' work of course consists of developing and providing formal training. Both the survey and the cases provide too little specific information on the nature of training efforts, to permit a conclusion more detailed than the general observation that 'traditional' training does have an important place in the strategies employed by the HRD professionals to achieve their objectives.

Of particular interest here are the initiatives to use training in such a way that it prepares workers for other forms of (informal) learning. The Belgian cleaning company ISS, for instance, provides certificates for employees who have received a certain amount of training, as a means to support the development of a learning culture, and 
more specifically to stimulate motivation for learning (see Chapter 4). This might seem contradictory to the objective of stimulating informal learning, but in fact it can be seen as a measure which gives employees who hold negative ideas on learning a positive feeling and more self-confidence. Both are prerequisites for using learning opportunities and sharing knowledge. So this measure can be seen as a stepping stone towards creating a learning culture and self-management in learning, even though it is associated with traditional training. Similarly, Alcatel Bell, a telecommunications company from Belgium, has introduced a minimum amount of training hours. More specifically, every employee is obliged to follow twenty hours of formal training, twenty hours of on-the-job training, and ten hours of Bell Permanent Training. This educational project should increase the employability of employees. Though at first glance it seems at odds with the general striving for increasing the use of informal learning opportunities, it can actually support such forms of learning. For example, it enables employees to rotate in different jobs, or because it increases their learning skills and self confidence in learning (see Chapter 4).

\section{Changing HRD practices}

A very interesting category of strategies is that of the interventions used to change HRD practices or HRD organisation.

These include initiatives to decentralise HRD activities or at least achieve a less centralised organisation of the HRD function. This can be seen as a means to distribute HRD tasks and responsibilities to managers and even employees, and to ensure that HRD professionals are in close contact with the organisation (which is considered essential for ensuring a link between HRD and the business). For instance, the German household equipment factory Bosch Siemens deliberately adopted a decentralised HRD strategy, despite the fact that the HRD department is a centralised function. Responsibility concerning many HRD activities is now transferred to local decisionmakers, who posses the necessary competencies and knowledge to judge which are necessary. This should serve to enhance the link between HRD and organisational needs (see Chapter 7).

Supporting management in HRD tasks and providing tools for HRD tasks, and increasing employee responsibility can be considered to be directed at the same purpose. In particular, providing models and methods for Personal Development Plans is an effective way for HRD professionals to transfer some HRD tasks to line managers and employees. In the British insurance firm Royal Scottish Assurance, HRD staff have designed and implemented monthly 'one-to-one interventions', which are development-oriented meetings between individuals and their manager. HRD provides documentation to support and to focus the meetings, and materials provided by the HRD department (such as a handbook where learning questions can be written down) support the personal development process (see Chapter 10). Such interventions are probably not always so much explicitly intended as strategies to implement HRD's new role, but can be considered as the actual manifestation of a new 'partnership in learning', shared between HRD practitioners, line management and workers. 
It is interesting to note that a small number of companies explicitly pay attention to developing skills of managers with regard to HRD. For instance, the Italian software company Datalogic has evaluated its managers to measure their ability and motivation to support employees in learning (see Chapter 8 ). Such measures clearly go beyond providing practical support for HRD tasks. An equally interesting point to note is that, in general, there appears little attention to the professional development of $H R D$ practitioners themselves. The German consulting firm GTZ was found to actively develop new skills in its HRD professionals in order to fulfil the new role of consultant and provider of support for line management (see Chapter 7). Since this new role is so radically different from the traditional roles of HRD professionals, such as developing and providing training, it might seem logical to expect that such a training would be beneficial for HRD professionals throughout more of the organisations. There is no evidence that the HRD professionals in the 27 other cases didn't work on their own professional development, it might be that more than one case engaged in such activities in this respect. However, it can be concluded that this is certainly not common. The survey results also did not indicate that professional development of HRD practitioners was a widely-used change strategy.

\section{Leading HRD strategies?}

Overall, it can be concluded that training is still an important HRD strategy, but it is complemented by strategies to support other types of learning (such as coaching, using information and communications technology (IC-T) to promote knowledge sharing etc.), and by activities meant to ensure a close link between HRD and business strategy.

HRD professionals not only invest in creating opportunities for informal learning, but also deliberately try to change attitudes towards learning, to ensure that these opportunities are actually used. For instance, methods to support informal learning are also sometimes used to change views of learning as a classroom activity. And sometimes even training-related measures are used to increase motivation for learning and boost learner self-confidence.

Though the case studies yielded a wide range of strategies employed by HRD professionals to realise their envisioned role, there was no clear indication of the relative weight of each of these strategies. The survey attempted to establish somewhat of a ranking order; which strategies are considered most important? This proved difficult, since nearly all strategies were rated 'relevant' or 'important' by respondents.Survey results did not indicate that great differences exist, but it does seem that some strategies are used more often than others. Most noticeable is the importance (still) attached to formal training programmes. In addition to newer methods for supporting learning (e.g. learning networks), formal training remains a learning mode of significant importance. Among the least often used strategies are instruments and initiatives to increase employee responsibility for learning. In general, however, differences are not very striking. Just as with general change strategies on the organisational level, HRD change strategies form a rich array in which not one strategy dominates. 
A related question asked in the survey was: do respondents expect the nature of HRD strategies, techniques and instruments to change in the near future? Which HRD strategies will become more important, and which will decline in use? Strikingly, respondents estimate that all strategies will become more important in the future. There is no immediate contrast between strategies in the field of training and in other areas (such as supporting informal learning or changing the HRD function). Though differences are relatively small, it is interesting to note that the limited number of strategies for which the difference between present use and expected use is relatively high, are using competence management and stimulating knowledge management. Both are currently not used very often, but it is expected that they will be implemented more often in the future. Nevertheless, differences are too small to attach much weight to these observations.

These results do not paint a picture of very innovative HRD practices, dominated by new initiatives such as knowledge management networks or stimulating a learning climate in the workplace. Of course, this is partly caused by the fact that HRD objectives are not that far-stretched (in other words: the envisioned role is not that ambitious). Providing training is still an important task (see the section on HRD's envisioned role in this chapter). On the other hand, these outcomes might indicate that HRD practices to some extent fall behind HRD visions. HRD professionals do want to broaden their horizon by also supporting more informal modes of learning, and by forming partnerships with managers and employees, but in practice, their work is still dominated by the more traditional, training related tasks. Of course, this is to be expected; new insights as a rule precede changing behaviours. Moreover, HRD professionals also have to deal with expectations from employees and managers regarding their own role, and the products they deliver. It is not possible to change overnight. But perhaps there are other factors influencing the change process? The next section examines this question.

One thing needs mentioning here. Just as there was little indication that companies looked for proof that working to become a learning organisation paid off, in terms of helping to achieve business objectives (see the section on organisational context, above), there were no signs that HRD professionals on a large scale sought to evaluate their contribution to the development, or lack thereof, of their company towards a learning organisation. Many HRD professionals do evaluate their training courses, but in only a few companies have they outlined a plan for evaluating their contribution on a more strategic level. There are exceptions, of course. Belgian telecommunications firm Alcatel Bell, for instance, collects statistics on job rotation (a means for informal learning) and on training hours. And Belgian insurance company DVV is using evaluation instruments on different levels in the organisation, such as organisational business plans, balanced score cards and competence management. By using these instruments, DVV also wants to measure whether HRD's role has changed successfully. But they realise that measuring the success of being a learning-oriented organisation is difficult and takes a long time (see Chapter 4).

Influencing factors

So, though HRD objectives indicate that HRD professionals are not only interested in providing training, but also seek to support other forms of learning, HRD practices 
reveal that traditional training is still very dominant. As a result, training related tasks are still very important in their total task load, next to newer tasks, such as consulting to line management on creating learning opportunities. And though HRD professionals feel it is important to share responsibility for HRD with line management and employees, they still carry most of the responsibility themselves (according to their own estimation).

It is worthwhile investigating whether there are specific reasons for this situation. Are certain specific influences keeping HRD professionals from changing their practices? Or is the current prevalence of training activities a desired result? This section presents the study's results concerning the practical change process, addressed by the third research question: what obstacles do European HRD departments encounter when trying to realise their new role, how do they overcome these constraints and which factors are conducive to the realisation of HRD's new role?

Both case study and survey results revealed a wealth of factors influencing the change process. For instance, rather fundamental issues such as employee motivation for learning, management's readiness to take on learning tasks, clarity of HRD's role, learning culture (or lack thereof) and more pragmatic issues such as time and money were all identified. Of particular interest is the finding that whereas in some organisations a highly motivated workforce was found to be a positive influence, other companies had to deal with the opposite; a lack of motivation for learning stifled change. In general, each conducive factor had an obstacle as its counterpart. Moreover, in many instances, firms mentioned one factor, e.g. management involvement, as both a conducive and an inhibiting force at the same time, because in some parts of the company managers were highly motivated and supported changes, whereas in other parts, managers were not so motivated and thereby held back changes. The survey was used as a means to investigate the general direction (positive, negative or neutral) of some of the most common factors affecting the change process. But it proved impossible to make any generalisations in this respect. It depends on the individual organisation what factors play a role, and what effect they have on the change process (positive or negative). Below, we will take a closer look at the obstacles and driving forces for changing HRD.

\section{Roadblocks to change}

Of the factors that sometimes hinder the realisation of HRD's new role, a very important one is a lack of motivation on the part of managers and/or employees to take on new learning tasks or to engage actively in learning processes. It is not clear from this research what might cause such a lack of motivation. Perhaps managers and employees are still used to a different way of working and don't want to give it up yet. Another possible explanation is a lack of faith in the concept of the learning organisation or in training in general. Barham and Rassam (1989) found that managers in companies that used to have a fragmented approach to training, sometimes do not view traditional courses as valuable learning experiences, or come to see training as a cost, rather than an investment for the future. Of course, it takes time to change such a pattern of expectations once it exists, especially when managers and employees still implicitly 
equate 'learning' to 'training'. Employees and managers may be waiting to see positive results of this new approach. But other factors, such as a shortage of time on the part of managers, lack of rewards for employees or little self-confidence when it comes to learning, could equally well play a role. Though the cause of low motivation or low sense of responsibility is unknown (and might be different for different organisations or even different managers and employees), it is clear that, if it occurs, it can hinder the realisation of a shared responsibility for HRD between management, employees and HRD professionals.

Low motivation for participating actively in learning might in some cases be linked to another possible constraint, namely a lack of clarity on HRD's role or lack of clarity on the need for learning. Either they find it difficult to establish their own role very distinctly, or (and?) they find it difficult to communicate this role in a clear and convincing manner to managers and employees. Whatever the case, it appears important to pay attention to this issue. How can HRD practitioners tempt managers and employees to become active partners in creating learning organisations? It is not unlikely that managers and employees are sometimes unmotivated to perform their new roles because it is not clear to them what is expected, why learning is relevant and what support HRD professionals will provide.

The third type of inhibiting factors might seem like 'stating the obvious'. Nevertheless, a lack of a learning culture was experienced as an inhibiting factor to realising HRD's intended role. Apparently, it is very difficult to create a learning culture, if such a culture doesn't already exist, at least partially. Most organisations in which this point was raised point to an insufficient learning culture in general, others narrow it down to insufficient knowledge sharing. This result indicates that it is very difficult to motivate employees to share knowledge or engage in learning processes if they are not used to this, or perhaps even show a reluctance to do so (this might indicate a relationship with the first category of objectives: lack of motivation for learning on the part of employees).

As well as these rather fundamental issues, pragmatic factors such as a lack of time for learning, and insufficient time or money to develop new HRD initiatives sometimes also influences the change process negatively.

One factor encountered quite often is a lack of time for learning on the part of employees. Work pressure is so high that it is hard to find time for learning. Of course, this compounds to the problem of lack of motivation and lack of a learning culture. The problem is that it creates a closed cycle; when people are continuously working very hard to keep up with new developments and changes (reactively), but are too busy to learn and reflect on existing practices, this makes it very hard to find solutions which might decrease the work load (pro-actively).

Some of the other more practical problems HRD professionals face concern their own role: scarce resources, too little time to develop new HRD initiatives, insufficient time to update materials or, in general, not enough HRD professionals. Of course the lack of time is associated with a scarcity of resources; if more money was available, extra human resources could be hired. Interestingly enough, there is no apparent relationship between the organisations that mention a lack of HRD professionals or lack of time, and the size of the HRD departments. Some of the organisations that 


\section{Saskia Tjepkema}

mention this problem employ only two HRD professionals, but it is also reported by HRD departments with a staff of 70 . So it seems that the cause for this problem lies more in the fact that realising the new role is very time-consuming, because at the outset it means more tasks for the HRD professionals (the new tasks as well as the traditional tasks, see the section on HRD's envisioned role, above). Perhaps more room will be created only when managers and employees have actually taken over some of the HRD tasks.

Regarding these pragmatic factors, it is interesting to consider a possible relationship with the outsourcing policy many companies adopted during the beginning of the 1990s. It is not certain whether this applies to many of this study's organisations, but during the last few years, many companies have cut back on their training budgets, and have chosen to hire or buy training rather than develop it in-house. It might be that some of the companies who are currently experiencing a lack of specialist practitioners have been through such an outsourcing process.

\section{Positive forces}

In addition to factors which obstruct innovation of the HRD function, all organisations could also point out positive influences on the change efforts. In many cases these are just the opposite of the constraints that sometimes hold back change.

Probably the most important conducive factor is active involvement, particularly from (top) managers (whereas low motivation for learning related tasks from this group was an important inhibiting factor). Sometimes HRD professionals find themselves dealing with both active and motivated managers and with those that are not motivated simultaneously. Employees with a high learning motivation were also mentioned as a conducive factor (whereas a lack of motivation for learning was experienced as a constraint). This leads to the impression that if a lack of motivation can be turned around into a strong involvement, this will strongly influence the change process in a positive way.

Clarity on HRD's new role can also be an important conducive factor, as can positive results of new HRD initiatives. These increase the organisation's motivation to change.

Another possible positive force is the existence of a learning culture, or a strong orientation towards innovation. If a company already has a corporate culture which is open to learning, this makes it easier to change HRD practices.

New organisational structures can provide employees with more possibilities for learning during work, which gives HRD professionals a good starting point to support work-related learning. This was also sometimes mentioned as a positive factor. For instance, the French transport company GT group mentions that its employees have more possibilities for learning due to increased contact with customers (Chapter 6). And the German chemicals producer AgrEvo and consulting firm GTZ identify that the introduction of teamwork and learning networks creates new learning opportunities (Chapter 7). So, whereas old organisational structures can hinder the adoption of a new HRD approach, the re-design of such structures can actually support them.

Finally, pragmatic factors can also play a positive role. Some organisations experienced such factors to be helping the change process. The most important of these are 
ample opportunities for training and development and/or sufficient HRD resources. Sufficient resources, or sufficient time for learning or for developing HRD materials, were not explicitly mentioned as conducive factors (though a lack of them was mentioned as an inhibiting factor). Thus, it seems that time and professional resources are frequently abundant

\section{Dealing with constraints and capitalising upon positive influences}

Some of the conducive factors might very well be necessary but insufficient conditions for firms to become learning-oriented organisations. Take 'time for learning' for instance. In situations where there is a fundamental lack of time for learning, which undermines change initiatives regarding, for instance, creating a learning culture, it is essential to find a solution for this problem. Even if other conditions are met, for instance HRD resources are plenty and senior management is highly committed to the concept of the learning organisation, until workload pressures are addressed, and time is made for learning, employees will continue to see learning as 'extra' to their daily work practices (which negatively influences their motivation for learning). It is not possible to provide advice that is valid in all situations, each organisation has to find out what are the necessary conditions that are lacking in their specific situation.

As the existence of conducive factors will usually prove to be not enough in itself to boost change, conversely, inhibiting factors do not necessarily preclude the achievement of becoming learning-oriented. If we take the same example, time for learning, in some firms, for instance Royal Mail, despite shift work and daily targets, time is being found to enable learning events to be scheduled in work time and in the work environment (see Chapter 10). These organisations have found effective strategies to cope with constraints.

The last question that was addressed in this research is how HRD functions cope with the roadblocks to charge (and/or fully use or stimulate the conducive forces). It was found that for many HRD departments, the general strategies employed to realise their intended role could be considered as the main 'coping strategies' (see the section on HRD strategies, above). However, some specific (extra) coping strategies were also encountered.

Communication seems to be the key word in these coping strategies. It is essential to make sure the organisation understands the (new) role of HRD, the intentions of the HRD department, what is expected of managers and employees (and why), the new vision on learning (broader than the old classroom approach) and has the necessary information on learning opportunites. This understanding and information is very important for managers and employees to be motivated to implement the changes and to bring about a fruitful partnership between HRD professionals, managers and employees.

More specifically, the following communication strategies were found:

- Communication directed at changing the view on (the need for) learning: For instance, it was found that all French case organisations pay attention to sharing knowledge and communication improvements in order to let employees know 
why change is necessary, why learning plays an important role in the change process of the organisation and that learning takes place not only by following courses, but also by learning from each other. If this communication succeeds in changing attitudes towards learning, they might serve to increase motivation for learning, as well as enhance the creation of a learning culture and promote knowledge sharing.

- Initiatives to increase communication or even co-operation with managers and employees to direct HRD initiatives more closely to their needs. For instance, the Italian software company Datalogic has adopted direct horizontal communication with line management as one of its main coping strategies. As is normal for middle-sized companies, new emergencies and changes are rather frequent. The HRD department deliberately adopts a very flexible approach and a listening attitude, which leads to teamwork between managers and HRD practitioners. One could say that the traditional distinction between line and staff is overcome. Such strategies can be expected to create goodwill and motivation. The Dutch building company KIBC found that improving communication and information flows are both very useful in decreasing resistance and increasing motivation for learning.

- Providing information on the position, roles and responsibilities of the HRD department, on learning needs and on learning possibilities. This might provide an antidote to the inhibiting factor of lack of information and might thus serve to increase motivation for learning and for actively participating in the renewed HRD function.

Other coping strategies were also found. These include:

- Measures to reduce the workload of HRD professionals, and thus tackle the problem of a lack of resource, such as recruiting new HRD professionals, delegating responsibilities to line managers, and providing a training programme for those managers and increasing efficiency of HRD work methods (a more clear definition of HRD roles and tasks to avoid double work).

- Measures which can be seen as efforts to underline the professional approach of the HRD professionals, and to make sure the organisation has a positive approach to the HRD function, which might increase the motivation to adopt new HRD practices, namely: showing added value of the HRD department, a careful planning of HRD activities and policy, and a critical evaluation of the effects of HRD.

- Efforts to ensure that a lack of learning possibilities does not hinder the change process, such as continuously updating the supply of HRD initiatives or increasing possibilities for knowledge sharing and informal learning.

- Strategies that are probably meant to increase motivation for learning in the workforce, by bringing in 'fresh blood', and making sure (through the recruitment process) that new workers are motivated for learning, application of new selection criteria in recruitment, and redeployment of some employees. 
- Activities that serve to provide the HRD professionals with a 'sounding board', and with new ideas for initiatives within their own companies, for instance, networking with other organisations that are implementing the learning organisation concept.

\section{Summary}

In summary then, this research points to changing conditions affecting businesses across Europe, and which lead to the formulation and implementation of new business strategies. Responding to the same conditions, and those new strategies, HRD departments and practitioners are developing new visions of their role. While success in achieving those visions is variable across the HRD departments studied in this project, and while it is also true to say that traditional training and development activities still figure prominently in HRD policy and practice, there are signs that significant changes occur, and will continue to occur, across Europe. Chapter 12 examines some of the implications of this changing scene. 